\title{
Preliminary Efforts Directed Toward the Detection of Craving of Illicit Substances: The iHeal Project
}

\author{
Edward W. Boyer • Rich Fletcher • Richard J. Fay • \\ David Smelson • Douglas Ziedonis • Rosalind W. Picard
}

Published online: 4 February 2012

(C) American College of Medical Toxicology 2012

\begin{abstract}
Many behavioral interventions, whether for the management of chronic pain, overeating, medication adherence, or substance abuse, are ineffective outside of the clinic or office environments in which they are taught. This lack of utility has spawned interest in enabling technologies that are capable of detecting changes in affective state that potentially herald a transition to risky behaviors. We have therefore undertaken the preliminary development of "iHeal", an innovative constellation of technologies that incorporates artificial intelligence, continuous biophysical monitoring, wireless connectivity, and smartphone computation. In its fully realized form, iHeal can detect developing drug cravings; as a multimedia device, it can also intervene as the cravings develop to prevent drug use. This manuscript describes preliminary data related to the iHeal Project and our experience with its use.
\end{abstract}

Keywords Drug abuse $\cdot$ mHealth $\cdot$ eHealth $\cdot$ Wireless communications $\cdot$ Drug craving $\cdot$ Craving $\cdot$ Mobile health

E. W. Boyer $(\bowtie) \cdot$ R. J. Fay

Department of Emergency Medicine,

University of Massachusetts Medical School,

55 Lake Avenue North,

Worcester, MA 01655, USA

e-mail: edward.boyer@childrens.harvard.edu

R. Fletcher $\cdot$ D. Smelson $\cdot$ D. Ziedonis

Department of Psychiatry,

University of Massachusetts Medical School,

Worcester, MA, USA

R. Fletcher $\cdot$ R. W. Picard

Media Laboratory, Massachusetts Institute of Technology,

Cambridge, MA, USA

\section{Introduction}

Behavioral modification interventions often fail in natural environments because patients have difficulty enacting them outside of the controlled setting of a clinic or office in which they are taught [1]. One reason for this failure is that patients neither detect biological and affective changes that herald increased risk, nor do they modify behaviors in a way that decreases health risk [1]. The shortcomings of behavioral interventions have spawned interest in technologies that are capable of detecting changes in affective state that herald transitions to risky behaviors such as substance use [1]. We have therefore initiated a series of efforts to develop "iHeal", an innovative constellation of technologies that incorporate artificial intelligence, continuous biophysical monitoring, wireless connectivity, and smartphone computation.

iHeal works in the following way (Fig. 1). Individuals with histories of substance abuse and PTSD wear a sensor band that measure electrodermal activity, body motion, skin temperature and, optionally, heart rate, each of which provides a measure of sympathetic nervous system activity due to arousal or stress [2]. The sensor band wirelessly transmits information to the smartphone, where a mobile software application monitors and processes the user's physiology data. When the software detects an increased level of sympathetic nervous system activity, iHeal assesses ecological and behavioral events surrounding the change in physiology. The assessment is simple and involves the iHeal software asking the user to annotate events by self-reporting affect, level of stress, drug cravings, activities, among other items. Collectively, these data - skin conductance, temperature, motion, time, location, and annotations - comprise a dataset that is used to train a machine learning algorithm to recognize behavioral transitions. The trained algorithm is downloaded onto the smartphone and the process repeated, 
with iterative periods of data collection, testing, and retraining improving the predictive ability of iHeal's algorithm.

The ultimate goal of iHeal is to identify in real-time drug cravings and deliver personalized, multimedia drug prevention interventions precisely at the moment of greatest need. While we ultimately hope to identify active drug craving and use from antecedent affective changes, iHeal requires a form factor that is highly acceptable to potential study populations. We therefore describe in this manuscript our preliminary efforts related to the iHeal Project and improving experience with its use: system architecture, identification of acceptable form factors, data encryption, and attrition of devices.

\section{Methods}

The Institutional Review Boards of every participating institution approved all phases of this study; all participants provided written informed consent and were compensated for their participation. We used a design approach to the iHeal Project; whereas the scientific method is analytic and grounded in hypotheses, a design approach is constructive $[3,4]$. Accordingly, we generated a system architecture for the iHeal Project that allows for (1) continuous detection of skin conductance, skin temperature, motion, and pulse; (2) machine learning software to identify when changes in physiology (e.g., increased pulse/skin conductance and motion) potentially arose from changes in sympathetic tone (as from drug craving and PTSD) rather than from exertion; (3) real-time annotations by users of affective states and activities in the time preceding drug use or cravings; and (4) a means to iteratively improve the ability of the machine learning program to identify craving/use episodes.

Once we had created the system architecture, we conducted a focus group to identify the preferred structure of the iHeal sensors and other operational features. We recruited patients at the Edith Nourse Rogers Veterans Administration Medical Center Domiciliary, a residential drug treatment unit. Using a combination of flyers and in-person presentations at community meetings, we sought to recruit Operation Iraqi Freedom and Operation Enduring Freedom-era veterans with co-occurring disorders of post-traumatic stress disorder and opioid abuse; we had no other inclusion or exclusion criteria. Opioid craving produces a more robust physiologic response than craving for drugs such as cocaine [5-9]. The focus group began with a broad description of iHeal, its purpose, and general functionality. Open-ended questions explored potential content; reflective listening, and summative statements encouraged elaboration. The interview then proceeded to explicit probes about the preferred location of sensor bands; the anticipated number of acceptable interactions between iHeal and user per day; and content for the annotation panel, such as intensity of drug craving, assessments of affect/stress, and activities. Results from our solicitation of user perspectives on materials allowed us to improve study components in preparation for the main study.

The mobile nature of the iHeal Project placed several unanticipated constraints on the investigation. The Veterans Administration (VA) assiduously protects the safety and confidentiality of all its patients, especially those participating in research investigations. Furthermore, recent security breaches have led the VA to adopt stringent security measures on mobile data storage devices. Although we intended to collect only de-identified data, the possibility existed for study participants to enter personal identifying information into other embedded smartphone programs such as text message or notetaking applications. We therefore encrypted all iHeal data files to ensure patient confidentiality; we also reprogrammed the smartphone to prevent data entry into other applications. An additional complication was loss of de-identified participant data if the iHeal smartphone was lost or sold. Although we conducted the study in a residential treatment center, we nonetheless created a detailed protocol for VA and press notification in the event a study device was lost.

\section{Results}

System Architecture The iHeal system architecture is presented in Fig. 1. Although multiple mobile computing platforms exist, we elected to use smartphones running Android OS 2.2 or later since this operating system supports the use of multiple Bluetooth connections from the sensor band to the phone. We selected Android over competitor operating systems because of its ease of programming and the ability to modify the appearance of the final product. Finally, the system architecture included, in addition to the mobile phone and sensor, a web server running on a desktop PC and a Microsoft SQL database running on a secure server in a high-security location. Software programmed into the mobile phone allowed three separate means to interact with the user: self-reported drug craving/stress initiated by the user; a random assessment and annotation, the timing of which was generated by the iHeal software; and an assessment and annotation that is triggered by changes in the user's physiology.

Focus Group Seven male veterans (six Caucasian, one African-American ages 27-55) undergoing residential treatment participated in the focus group. All members had substance abuse disorders including opioid abuse, as well as post-traumatic stress disorder. Despite being homeless and in treatment for problematic substance use, all participants owned mobile phones with active service plans; three (42\%) 
Fig. 1 iHeal system architecture

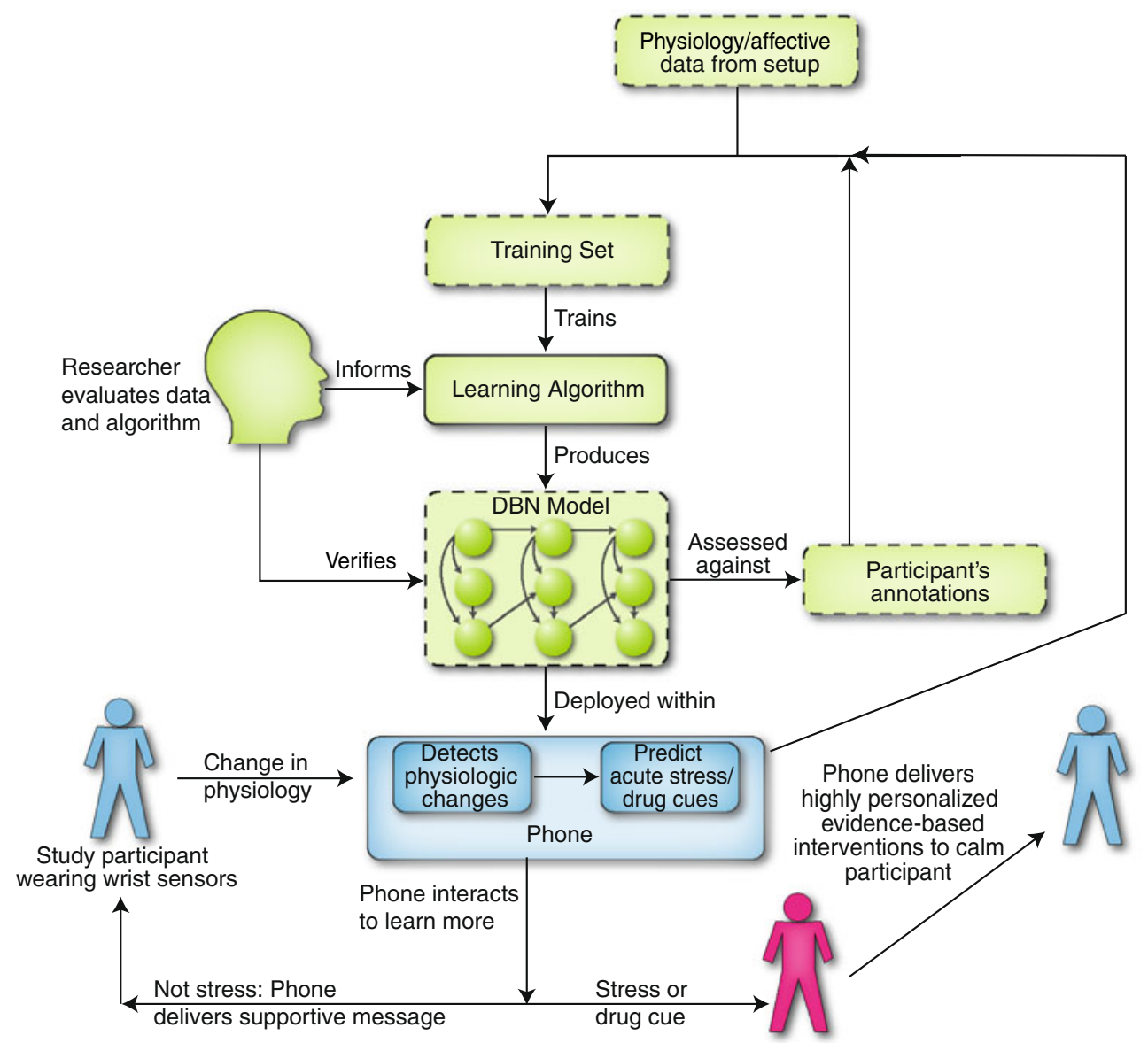

owned smartphones with touch screens. Those with smartphones indicated that they would never return to the use of flip phones (also called "feature" phones) as the older devices lacked desirable functionality. While they appreciated the immediacy of contact of text-based messages, participants found the potential for inadvertent misunderstandings between individuals and elimination of personal contact as disadvantages of text messages. Rather than receive text messages to promote treatment protocols, participants reported that videos, apps, games, calming songs, or other media would be preferred methods for introducing behavioral interventions.

The sensors measuring skin conductance, pulse rate, and acceleration that we presented to the focus group were about the size of a half dollar coin and housed in a standard athletic wristband. Focus group members indicated that while this embodiment was acceptable for research purposes, wearing it outside of a residential drug treatment center might attract unwanted attention. The focus group therefore recommended that a more robust and less stigmatizing version would be necessary before the device could be worn in public. Specific comments were to develop a waterproof version, a sensor band that had the appearance and functionality of a wristwatch, or a sensor band that was worn on the ankle.

\section{Discussion}

Mobile health, also known as "mHealth," is the use of mobile computing devices to support patient health [10]. At present, most mHealth applications focus upon tracking self-reported health indicators (e.g., weight) or the sharing of information; via existing services such as $3 / 4$ G networks, patients can exchange information or seek support from health-related usergroups [11]. While such interactivity

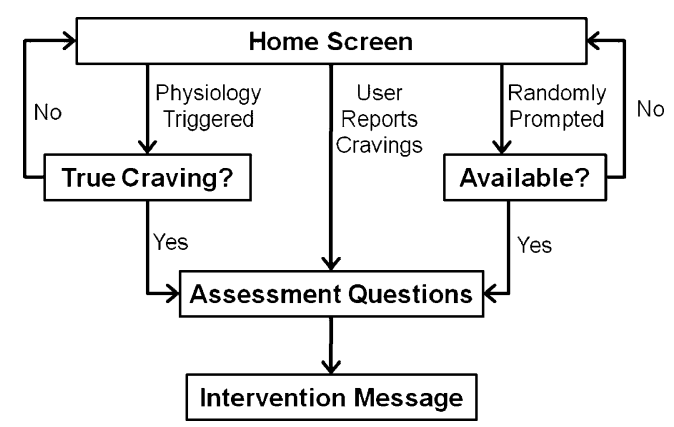

Fig. 2 iHeal state diagram demonstrating the number of ways that users can interact with the enabling technology 
may be useful among a group with a nonstigmatizing disease, hidden populations - such as persons who abuse illicit drugs, have psychiatric conditions, or who are infected with HIV - may not openly share information regarding their condition. Stigmatized patients, who still receive much of their care from face-to-face interactions with clinicians, may therefore benefit from the emerging branch of mHealth known as enabling technologies [12]. Enabling technologies such as iHeal differ from existing healthcare applications in that they may to incorporate biosensors that potentially allow the delivery of healthcare precisely at the moment of greatest need. The monitoring functions and ubiquitous presence of enabling technologies can prevent rather than manage poor health by truncating the adverse behaviors that lead to many common, expensive, and intractable medical conditions.

Because enabling technologies are intended to be a pervasive presence, developing usable interfaces with designers and form factors tuned to user preferences are critically important to their acceptance [12]. The several ways in which a user can interact with iHeal (Fig. 2) place the technology at risk of becoming an annoying pest. Acceptability, therefore, hinges on correct timing of interactions between iHeal and its user, the skill with which iHeal communicates, and how well interventions resonate with the recipient. Our focus group results indicate that selfidentified interventions for drug craving are more acceptable than the standard approach of using text message-based content couched in cognitive behavioral theory that have been developed by treatment experts. By extension, these findings suggest that study designers should rely upon recipients rather than "experts" to create intervention content that would be maximally effective at preventing relapse. The use of customized interventions may seem an intuitive strategy, but this approach in uncommon in behavioral science research even though interventions self-selected by the patient under treatment have been recognized as a potentially valuable treatment for problematic substance use [13]. Furthermore, the use of customized interventions dovetails well with iHeal, which is designed to recognize physioaffective states and apply an intervention early to disrupt the conditioned responses through maximizing coping-self statements and self-efficacy - the essence of cognitive behavioral theory.

Considering that wrist sensors have a long history in physiometric research, are easy to manufacture, and are contained in an unobtrusive housing, we were surprised by the lack of enthusiasm for this form factor. The heightened visibility created a potential for stigmatization that, in the opinion of our focus group members, outweighed user convenience and contributed to their poor acceptance. Alternative form factors such as sensors worn on the ankle carry some disadvantages. First, electrodermal activity is highly dependent on body location, and measurement of physiologic responses at the ankle is poorly understood. Considerable laboratory work to measure individuals' responses to drug use cues (e.g., material such as drug paraphernalia designed to elicit a craving response) must therefore precede iHeal field testing. Second, ankle sensors can simply fall off during normal activity without the user's knowledge, a drawback to be confronted by improved sensor band design.

Cellular phones are prone to loss, and using smartphones as mobile data storage devices increases the risk for losing not only research data, but also personal information as well. Although we adapted the smartphones' functionality to eliminate the possibility of storing personally identifiable data, we incorporated several additional protections. First, all smartphones required password entry before the device would activate. Second, we limited potential hacking of the wireless signal by using Bluetooth communications in nondiscoverable mode and by limiting the power output on devices so that the effective communication range for sensor was several meters. Finally, we encrypted files on study computing devices by using cryptographic modules conforming to a FIPS 140-2 standard [14].

The iHeal smartphone represents a potential cash equivalent to individuals with histories of drug abuse through theft and subsequent sale. Even though we avoided this problem by conducting our investigation in residential drug treatment, we do not believe that the diversion of study devices to illicit sale will occur since multiple NIDA-funded investigators have provided cellphones and PDAs to active drug abusers who participated in a demanding regimen of randomly timed interruptions $[15,16]$. Participants were compensated for the return of devices, a practice that prevented attrition of study devices $[15,16]$.

This study demonstrates that conducting clinical trials using enabling technologies in natural environments will require a deeper understanding of user preferences. In particular, a focus on preventing identification of research participants may prevent subsequent stigmatization. Technical issues related to data security that are well described should be implemented to ensure the confidentiality and anonymity of study participation. Finally, attrition of expensive mobile data collection platforms such as smartphones can be prevented through selection of study site as well as remuneration for return of devices.

Acknowledgments This research was supported by NIH ARRA grant RC1-DA028428.

\section{References}

1. Boyer EW, Smelson D, Fletcher R, Ziedonis D, Picard R (2011) Wireless technologies, ubiquitous computing, and mobile health: application to drug abuse treatment and compliance with HIV therapies. J Med Toxicol 6:212-216 
2. Boucsein W, Schaefer F, Sommer T (2001) Electrodermal longterm monitoring in everyday life. In: Fahrenberg JMM (ed) Progressin ambulatory assessment. Hogrefe, Gottingen, pp 549-600

3. Cross N, Naughton J, Walker D (1981) Design method and scientific method. Des Issues 2:195-201

4. Cross N (2001) Designerly ways of knowing: design discipline versus design science. Des Issues 17:49-55

5. Heather N, Stallard A, Tebburr J (1991) Importance of substance cues among heroin users: comparison of two methods of investigation. Addictive Behavior 16:41-49

6. Zhong F, Wu L, Han J (2006) Suppression of cue-induced heroin craving and cue-reactivity by single-trial transcutaneous electrical nerve stimulation a $2 \mathrm{~Hz}$. Addict Biol 11:184-189

7. Smelson D, Kilker C, Starosta A, Losonczy M (2002) An analysis of cue-reactivity among individuals with schizophrenia compared wtih cocaine addicts without schizphrenia. Psychiatr Serv $53: 1612-1616$

8. Smelson D, Williams J, Ziedonis D, Losonczy M, Kaune M (2006) The efficacy of olanzapine for decreasing cue-elicited craving in individuals with schizophrenia and cocaine dependence: a preliminary report. J Clin Psychopharm 26(1):9-12

9. Smelson D, Roy A (1997) Resperidone diminished cue-elicited craving in withdrawn cocaine-dependent patients. Can J Psychiatr 42:984
10. Istepanian R, Jovanov E, Zhang Y (2004) Introduction to the special section on mHealth-beyond seamless mobility and global wireless health-care connectivity. IEEE Trans Inf Technol Biomed 8:405-409

11. Fox S (2011) The social life of health information. Pew Research Center, Washington

12. Haux R, Geitzelt M, Hellrung N et al (2009) Health-enabling technologies for pervasive health care: a pivotal field for future medical informatics research and education. In: Adlassnig K (ed) Medical Informatics in a United and Healthy Europe. European Federation for Medical Informatics, Copenhagen, pp 14-16

13. (2002) Crano W, Burgoon M (eds) Mass media and drug prevention. Classical and Contemporary Theories and Research. Lawrence Erlbaum Publishers, Mahwah, NJ

14. (2001) Security requirements for cryptographic modules. In: National Institute of Standards and Technology DoC (ed) Gaithersburg, MD

15. Freedman M, Lester K, McNamara C, Milby J (2006) Cell phones for ecological momentary assessment with cocaine-addicted homeless patients in treatment. J Subst Abuse Treat 30:105-111

16. Epstein D, WillnerReid J, Vahabzadeh M, Mezghanni M, Preston K (2009) Real-time electronic diary reports of cue exposure and mood in the hours before cocaine and heroin craving and use. Arch Gen Psychiatry 66:88-94 